\title{
Telemedicine Intervention Efficiency on Mobility Recovery After Bariatric Surgery: The MyGoodTrip Randomized Controlled Trial
}

\section{Mathieu Bruzzi}

Hopital Europeen Georges Pompidou

Claire Carette ( $\nabla$ claire.carette@aphp.fr)

Assistance Publique - Hopitaux de Paris

Katia Lurbe i Puerto

Hopital Europeen Georges Pompidou

Tigran Poghosyan

Hopital Europeen Georges Pompidou

Marion Bretault

Hopital Ambroise-Pare

Aurélie Vilfaillot

Hopital Europeen Georges Pompidou

Gilles Chatellier

Hopital Europeen Georges Pompidou

Jean-Marc Chevallier

Hopital Europeen Georges Pompidou

Sébastien Czernichow

Hopital Europeen Georges Pompidou

Claire Rives-Lange

Hopital Europeen Georges Pompidou

\section{Research}

Keywords: MyGoodTrip, Telemedicine, mobility recovery, bariatric surgery

Posted Date: August 28th, 2020

DOI: https://doi.org/10.21203/rs.3.rs-58886/v1

License: (c) (i) This work is licensed under a Creative Commons Attribution 4.0 International License.

Read Full License 


\section{Abstract}

Background: The use of telemedicine may improve mobility recovery in patients with severe obesity undergoing bariatric surgery. Our aim was to evaluate a telemedicine intervention program dedicated to the promotion of physical activity including teleconsultation and telemonitoring on patients' number of steps in the six first months following bariatric surgery.

Methods: This study was an open label randomized controlled trial. Patients were included during the first week after bariatric surgery. Patients were randomized in two groups of telemedicine intervention: (i) physical coaching focusing on mobility (=TelePhys group) or (ii) dietary coaching (=TeleDiet group). The primary outcome was the difference in the delta number of steps measured during a period of 14 days at the first and sixth postoperative months between the TelePhys and the TeleDiet groups. Data were collected using the connected wireless watch pedometer. Body weight evolution and health-related quality of life were also evaluated. An additional qualitative approach was conducted to evaluate patients' perception of the connected devices used in the trial.

Results: Ninety patients with mean age (SD) 40.6 years $( \pm 10.3)$ were included. Seventy-three patients were females $(81.1 \%)$ and 62 had gastric bypass $(69 \%)$. The two intervention groups were comparable at inclusion except for the number of steps significantly higher in the TelePhys group $\left(1073.10^{2} \pm 471.10^{2}\right.$ versus $839.10^{2} \pm 437.10^{2}, p=0.045$ ). An increase of the mean number of steps between the first and the sixth month was found in both groups but this delta was significant only in the TeleDiet group $(p=0.010)$. No difference was found when comparing the delta between the two intervention groups. A significant increase in quality of life was observed in both groups without any significant differences between the two interventions.

Conclusions: Our study was not able to show a significant superiority of a telemedicine intervention dedicated to physical activity in mobility recovery after bariatric surgery. The early postoperative time frame for our intervention may explain the null findings. Further research will need to focus on long-term interventions.

Trial registration: This study was registered the $23^{\text {th }}$ of March 2016 (ClinicalTrials.gov Identifier: NCT02716480).

\section{Introduction}

During the last decades, bariatric surgery proved to be the most effective treatment of morbid obesity in the long-term [1-4]. However, a third of patients regain weight, after the first year during the following bariatric surgery, compromising the health benefits achieved with surgery [4-8]. Weight regain is due to an inadequate follow-up with poor compliance to dietary or physical activity advices and sometimes, an insufficient preparation before surgery. Moreover, early recovery to physical activity is a well-known success factor after bariatric surgery [9-13]. Gould et al. showed that over two third of patients miss appointments during the two first postoperative years and only $40 \%$ had their four annual recommended 
follow-up visits during the first year following gastric bypass surgery [14]. Interestingly, some studies showed that the most important key factor to affect aftercare is the distance of patient's home from urban areas $[14,15]$. In order to improve long-term results and follow-up after surgery, the use of telemedicine or eHealth has been proposed for delivering healthcare to operated patients [11,15-18]. The concept consists in the delivery of health-related services and information using telecommunication technologies or digital devices. The beneficial value of telemedicine as a unique postoperative care is not yet established, but its association to standard care could improve clinical outcomes. A recent review by Coldebella et al. [16] analyzing the use of telemedicine showed that the available literature is poor, but that it improved some psychiatric disorders without significant impact on weight loss and quality of life [16]. The two RCTs included in this review did not report any data on physical activity.

The aim of our study was to evaluate a telemedicine intervention program dedicated to the promotion of physical activity including teleconsultation and telemonitoring in the six first months following bariatric surgery.

\section{Methods}

This study was conducted in two university teaching public hospitals in Paris area (France). It employed a mixed-methods design [19] based on an open label randomized controlled trial that integrated quantitative data collected by questionnaires and qualitative data through focus groups and in-depth individual interviews using a sequential "QUANT -> quali" temporality design (the quantitative component precedes the qualitative one and it is predominant). According to Creswell and Plano Clark (2011)'s classification [20,21], this study combined triangulation (to converge results from different methods in order to validate interpretations) and complementarity (to reinforce, illustrate or clarify the results of one method by those of another). Moreover, it applied an interpretative strategy for integrating the quantitative and qualitative data collected. This study was approved by the institutional medical ethical board (CPP 15053). This study was registered with the ClinicalTrials.gov identifier: NCT02716480. The sponsor was My Goodlife SAS (Paris, France).

\section{Patients' inclusion}

Patients who underwent bariatric surgery (gastric bypass, sleeve and adjustable gastric band) were included from June, 2016 to July, 2018. Indications to perform surgeries relied on the guidelines published by the National Institute of Health [22]. Patients were included in the study during the first week postoperatively and were followed for 6 months. Telemedicine interventions began one month postoperatively. Inclusion criteria were as follows: age $>18$ years, a preoperative weight less than $150 \mathrm{~kg}$ (due to technical weight limit of the scale ), primary surgery only, no postoperative complication between surgery and 1 month post-operative, a valid home web access with computer, tablet or smartphone, a signed informed consent, ability to read and write French language and a valid affiliation to the French social security. All patients who did not meet the inclusion criteria were excluded. At inclusion, the 
informed consent was signed and the medical team gave two wireless telemonitoring devices to the patient: a connected body weight scale and a pedometer band $\left(\right.$ Terraillon $^{\odot}$ ).

\section{Randomization and allocation}

The randomization was computer-generated and allocation of patients was generated after the patients had signed the informed consent. Patients were randomized in two different and parallel intervention groups including telemedicine: (i) remote physical coaching by phone led by a mobility coach (=TelePhys group) or (ii) remote dietary coaching by phone led by a dietitian according to standard French guidelines [22] (=TeleDiet group). In the TelePhys group, the web platform allowed self-administration of a questionnaire to establish patient's diagnosis of mobility to enable personalized coaching. All coaching teleconsultations lasted 20 to 30 minutes and were repeated monthly during the six-month period of the study. For both intervention groups, the web platform tracked the parameters collected by connected devices (pedometer and connected scale), organized appointments with patients and ensured the traceability of exchanges with patients.

\section{Primary outcome measure: number of steps}

The primary outcome was the difference in the delta number of steps measured during a period of 14 days at the first and sixth postoperative months between the TelePhys and the TeleDiet groups. Data were collected using the connected wireless watch pedometer.

\section{Secondary outcomes}

Weight change was analyzed during the 6 postoperative months using the connected weight scale and reported as weight in kilograms $(\mathrm{Kg})$ and percent of weight loss (\%WL). Health-related quality of life was measured at baseline and 6 months postoperatively using the Quality Of Life, Obesity and Dietetics (QOLOD) scale analyzing five categories as physical impact, psychosocial impact, sex life, comfort with food, and dieting experience [23,24].

Focus groups and individual interviews were conducted to qualitatively illustrate the patient's experience through the use of connected body-weight scale and watch pedometer, their eating practices and physical activity behaviors after bariatric surgery. Drafts of the interview guides were revised accordingly after review by researchers and clinicians who worked with bariatric patients. Interviews items were structured into 3 major thematic areas: (i) social representations on healthy eating practices and physical activity, (ii) social conditions for lifestyle changes, adaptation or inertia and, (iii) assessment on how health digital devices and telemedicine helped them to implement nutritional prescriptions. Questions covered a range of topics on dietary and physical activity behaviors, weight loss, and surgical experiences. Participants were also asked to compare their pre-surgery and post-surgery experiences and to identify the factors and conditions that induced changes in their own thoughts, attitudes and behaviors.

\section{Statistical analyses}


The results are reported according to the criteria of the Consort Statement. An intention-to-treat analysis was performed. Quantitative and qualitative variables were described by means (SD) and frequencies (\%). We tested for potential differences using Anova or Kruskall-Wallis test for continuous variables and Fisher Exact test for discrete variables. Paired t-test was used for intra-group comparison. Data analyses were conducted using SAS version 9.4 (SAS Institute) and $p<0.05$ was considered to indicate statistical significance.

Concerning qualitative analyses, individual and group interviews were audio-recorded and transcribed verbatim. We performed a thematic content analysis with the support of the software ATLAS.ti 8.2.4 (ATLAS.ti.GmbH), coding the data with the constant comparative technique [25] around the three major thematic areas (social representations, social conditions for lifestyle changes and assessment on ehealth devices for nutritional prescription adhesion) and cross-checking for accuracy. Quotes in this paper were selected based on conceptual fit and according how they capture the common experiences expressed by more than one interviewee.

\section{Results}

\section{Patients}

Ninety participants were included in the study. Mean (SD) age was $40.6( \pm 10.3)$ years and BMI was 43.3 $( \pm 5.6) \mathrm{kg} / \mathrm{m}^{2}$, with a vast majority of women $(n=73 ; 81 \%)$. The most performed surgery was gastric bypass $(n=62 ; 69 \%)$, followed by gastric lap band $(20 \%)$ and sleeve $(11 \%)$. Preoperative characteristics and type of surgery performed in the TelePhys and the TeleDiet groups are detailed in Table 1. The two intervention groups were comparable at inclusion. Among the ninety patients enrolled, 70 of them completed the study until the sixth month i.e. $22.2 \%$ of lost to follow-up. Between the patients in TelePhys group ( $n=38)$ and TeleDiet group $(n=32)$ who completed the study, we obtained 14-day number of steps from the connected pedometer watch during the first and the sixth month in $23(60.5 \%)$ and 20 (62.5\%) patients respectively. Flow-chart of the study is presented in Figure 1.

\section{Primary end-point}

Concerning the number of steps, the two intervention groups presented significant difference in the 14day period step numbers at the beginning of the study during the first month monitoring with a superiority for the TelePhys group $\left(1073.10^{2} \pm 471.10^{2}\right.$ versus $\left.839.10^{2} \pm 437.10^{2}, p=0.045\right)$ (Table 2). There was no more significant difference between the two groups at the sixth month monitoring. An increase of the mean number of steps between the first and the sixth month was found in both the TeleDiet (delta $=338.10^{2} \pm 512.10^{2}$ ) and TelePhys (delta $=142.10^{2} \pm 535.10^{2}$ ) groups but this delta was significant only in the TeleDiet group $(p=0.010)$. Moreover even if the delta tended to be more important in the TeleDiet group, no difference was found comparing the delta between the two intervention groups $(p=0.240)$. 
Using all the data concerning day steps monitored by the connected band pedometer, Figure 2 represents the increase of the mean day steps in both groups. A peak of physical activity was seen monthly in each intervention group corresponding to the days around coaching teleconsultation.

\section{Secondary end-points}

\section{Weight change monitoring with the connected weight scale}

As expected, a significant weight loss was observed with bariatric surgery for both intervention groups at the sixth month (Table 3). No significant difference was found between groups concerning the delta of weight loss at the end of follow-up. The Figure 2 illustrates the decrease of the mean body weight in both groups using all the data monitored by the connected weight scale.

\section{Quality of life}

The overall QOLOD scale increased between baseline and the $6^{\text {th }}$ postoperative month was assessed in 21 patients of both TeleDiet and TelePhys groups. Each categories as "physical impact", "psychosocial impact", "sex life", "comfort with food", "dieting experience" were significantly improved at the end of the follow-up; however, no significant differences were found between the TeleDiet and TelePhys groups (Table 4).

\section{Experiences on e-health}

For the qualitative study, a total of 18 participants were interviewed. Among the 10 patients from the TeleDiet group, two had an individual interview (2/10) and eight participated to focus group (8/10). Among the 8 patients from the TelePhys group, four had individual interview (4/8) and four participated to a focus group (4/8). Interviews were conducted by a sociologist (KLP) and lasted for about 60 minutes for the individual ones and between 150 and 200 minutes for the focus groups.

Interviewees from both groups considered telemedicine as a helpful complementary tool along their postsurgery follow-up to strengthen their adherence to nutritional and physical recommendations. Four major reasons were expressed: 1) "The regular contacts, in the very context of my daily life, with the coach helped me to keep motivated in the efforts I needed to make in order to change my eating habits and start moving more", 2) "Having an expert with whom to speak regularly by phone about my situation was reassuring", especially because "even if medical team had previously informed us about the weight loss process and food changes following surgery, these are actually hard to go through once back home", 3) "The coach asked me for what I was doing, in my real life; so we could work together on how to customize" and 4) "It facilitates near-immediate responsiveness to individual problems and difficulties" (basically related to the rapid and intense weight loss and erroneous beliefs or incomplete food information) in order to achieve the expected behavioral changes.

Interviewees hold differing views on the usefulness of the digital health devices. Self-tracking in present time allowed them to objectify the physical changes achieved and to get benchmarks in the effort to be 
provided. However not all participants were proved to be equal as regards to digital competences. Indeed, the one less familiar with New Information and Communication Technologies (NICT) who could get some help from their children made a passive use of the devices: more as a data to feed research than an information to improve their own process of behavior change.

Whereas interviewees from the TeleDiet group were all satisfied with the intervention, those from the TelePhys group would have rather choose a dietary counselling as they considered it was too early for them to get involved in physical activity. Despite their willingness, all interviewees expressed difficulties in installing higher and sustained physical activity in the very context of their day-to-day lives. Adherence of physical activity advice not only met professional and family constraints, but especially the lack of transport and leisure infrastructures which could enable them to increase their walk or cycling time in a daily base. The qualitative study identified three major factors that jeopardize patients' efforts to achieve their physical activity goals, namely: 1) lack of urban policies that would promote outdoor activities with either practicable green areas, safe pedestrian walkways and bike path network, and invest in sport infrastructures accessible for everyone, 2) the long Paris-suburban areas of home-to-work travel time and 3) extended working hours.

\section{Discussion}

This study is the first randomized controlled trial (RCT) comparing the postoperative effect of physical activity coaching by telemedicine in patients who had undergone bariatric surgery, compared to dietary advices by telemedicine. An increase of the mean number of steps between the first and the sixth month was found in both groups but this delta was statistically significant only in the TeleDiet group $(p=0.010)$ and finally no statistical difference was found comparing the delta between the two intervention groups. A significant increase in quality of life was observed in both groups without any significant differences between the two interventions

Overall, the present study proved that connected telemedicine with physical activity measurement was feasible and acceptable, with a $77 \%$ rate of participants who had completed the 6-month follow-up period despite technological problems with the connected devices. Indeed, $22 \%$ of participants stopped the study mostly because of equipment dysfunctions.

On the other hand, results of the available literature suggest that the use of telemedicine has the potential to improve the basic knowledge of bariatric surgery patients in the preoperative period, and also to support patients in changing their lifestyle after surgery $[11,16,18]$. The results of these studies $[11,16,18]$ were supportive of telemedicine for providing health services in patients undergoing bariatric surgery. Coldebella et al. [16] also showed that only two randomized controlled trials are available on this topic and that telemedicine in association with standard dietary advices improved eating psychiatric disorders, but did not impact weight and/or quality of life changes. As no data existed on the impact of telemedicine on physical activity from RCT conducted in patients going through bariatric surgery, our study was conducted to compare the postoperative effect of telemedicine with dedicated physical activity 
coaching on patient's mobility, illustrated by the number of steps prospectively collected via connected wireless pedometer (Terraillon $\odot$ ). Interestingly, we did not find any effect on the total number of steps, nor on weight loss and quality of life in comparison to standard dietary advice using telemedicine as well. As the qualitative study brought in, professional and family constraints and lack of urban and transport policy promoting physical activity may help to partly explain our null results.

A question persists: which process implicated in physical activity changes can be used to motivate patients to adopt a more active lifestyle after bariatric surgery? A first hypothesis is that the addition of a targeted physical activity coaching in the first six months following surgery cannot be efficient because of strong and significant impact of weight loss during this time frame. During this postoperative period, patients live a sort of "honeymoon" and weight loss, associated with standard dietary advices, are sufficient enough to obtain the expected results. Previous studies support this theory and have shown that the strong physiological effects of surgery during the initial weight loss phase were the most significant reasons for lack of significant effect of behavioral treatment $[6,15,26,27]$.

Moreover, it is now established that the earliest process of change in mobility is "raising awareness". This theoretical aspect is really interesting but "raising awareness" in a chronic disease such as obesity cannot be dissociated with the notion of time. Physical coaching via telemedicine for operated patient needs to be analyzed with a more longer follow-up, in order to remove biases created by the immediate and impressive effect of weight loss. Long-term physical coaching associated with standard advices and remotely delivered interventions must have an interest at the end of the weight loss phase for maintaining a satisfying physical activity level for weight regain prevention.

The strengths of this study are illustrated by the fact that it represents the first multicentric connected RCT achieving results on physical activity, with acceptable follow-up, and because it uses a complementary qualitative approach to reinforce, illustrate or clarify results. Mixed methods research helps capture the complex influences of individual and contextual processes [28]. Limitations include the short follow-up and the small sample of the qualitative study which may preclude the drawing of any definitive conclusions.

To conclude, our study was not able to show a significant superiority of a telemedicine intervention dedicated to physical activity in mobility recovery after bariatric surgery. The early postoperative time frame for our intervention may explain the null findings. Further research will need to focus on long-term interventions.

\section{Declarations}

\section{Funding}

This study was funded by the public Agence de l'Environnement et de la Maîtrise de l'Energie (ADEME).

\section{Acknowledgments}


We would like to thank Audrey Chartrin and Alizée Padieri for their help in conducting the teleconsultations.

\section{Conflict of Interest}

SC is a co-founder of MyGoodLife. The other authors declare that they have no conflict of interest.

\section{Ethical Considerations}

All procedures performed in studies involving human participants were in accordance with the ethical standards of the institutional and/or national research committee and with the 1964 Helsinki Declaration and its later amendments or comparable ethical standards.

\section{Informed Consent}

Informed consent was obtained from all individual participants included in the study.

\section{References}

1. Sjöström L, Lindroos A-K, Peltonen M, Torgerson J, Bouchard C, Carlsson B, Dahlgren S, Larsson B, Narbro K, Sjöström C D, Sullivan M and Wedel H 2004 Lifestyle, diabetes, and cardiovascular risk factors 10 years after bariatric surgery. vol 351

2. Sjöström L 2013 Review of the key results from the Swedish Obese Subjects (SOS) trial - a prospective controlled intervention study of bariatric surgery. J. Intern. Med. 273 219-34

3. Sjöström L, Gummesson A, Sjöström C D, Narbro K, Peltonen M, Wedel H, Bengtsson C, Bouchard C, Carlsson B, Dahlgren S, Jacobson P, Karason K, Karlsson J, Larsson B, Lindroos A K, Lönroth H, Näslund I, Olbers T, Stenlöf K, Torgerson J and Carlsson L M 2009 Effects of bariatric surgery on cancer incidence in obese patients in Sweden (Swedish Obese Subjects Study): a prospective, controlled intervention trial Lancet Oncol. 10 653-62

4. Sjöström L, Narbro K, Sjöström C D, Karason K, Larsson B, Wedel H, Lystig T, Sullivan M, Bouchard C, Carlsson B, Bengtsson C, Dahlgren S, Gummesson A, Jacobson P, Karlsson J, Lindroos A-K, Lönroth H, Näslund I, Olbers T, Stenlöf K, Torgerson J, Agren G and Carlsson L M S 2007 Effects of bariatric surgery on mortality in Swedish obese subjects. N. Engl. J. Med. 357 741-52

5. Adams K F, Schatzkin A, Harris T B, Kipnis V, Mouw T, Ballard-Barbash R, Hollenbeck A and Leitzmann M F 2006 Overweight, obesity, and mortality in a large prospective cohort of persons 50 to 71 years old. N. Engl. J. Med. $355763-78$

6. Sarwer D B, Wadden T A, Moore R H, Baker A W, Gibbons L M, Raper S E and Williams N N 2008 Preoperative eating behavior, postoperative dietary adherence, and weight loss after gastric bypass surgery Surg. Obes. Relat. Dis.

7. M. D, D.J. R, J.J. C, L. M, B. S, L. O-R, N. R, S. Y, M. F, W.B. I and M. B 2010 Re-emergence of diabetes after gastric bypass in patients with mid- to long-term follow-up Surg. Obes. Relat. Dis. 
8. Christou N V., Look D and MacLean L D 2006 Weight gain after short- and long-limb gastric bypass in patients followed for longer than 10 years Ann. Surg.

9. Yen HY and Chiu HL 2019 The effectiveness of wearable technologies as physical activity interventions in weight control: A systematic review and meta-analysis of randomized controlled trials Obes. Rev. $201485-93$

10. Baillot A, Audet M, Baillargeon J P, Dionne I J, Valiquette L, Rosa-Fortin M M, Abou Chakra C N, Comeau E and Langlois M F 2014 Impact of physical activity and fitness in class II and III obese individuals: A systematic review Obes. Rev.

11. A. B, P. B, M. T and M.-F. L 2017 Feasibility and effect of in-home physical exercise training delivered via telehealth before bariatric surgery J. Telemed. Telecare

12. Herring L Y, Wagstaff $C$ and Scott A 2014 The efficacy of 12 weeks supervised exercise in obesity management Clin. Obes.

13. Bond D S, Vithiananthan S, Thomas J G, Trautvetter J, Unick J L, Jakicic J M, Pohl D, Ryder B A, Dean Roye G, Sax H C and Wing R R 2015 Bari-Active: A randomized controlled trial of a preoperative intervention to increase physical activity in bariatric surgery patients Surg. Obes. Relat. Dis.

14. Gould J C, Beverstein G, Reinhardt S and Garren M J 2007 Impact of routine and long-term follow-up on weight loss after laparoscopic gastric bypass Surg. Obes. Relat. Dis.

15. Bradley L E, Forman E M, Kerrigan S G, Goldstein S P, Butryn M L, Thomas J G, Herbert J D and Sarwer D B 2017 Project HELP: a Remotely Delivered Behavioral Intervention for Weight Regain after Bariatric Surgery Obes. Surg.

16. Coldebella B, Armfield N R, Bambling M, Hansen $\mathrm{J}$ and Edirippulige $S 2018$ The use of telemedicine for delivering healthcare to bariatric surgery patients: A literature review J. Telemed. Telecare 24 $651-60$

17. Versteegden D P, Van Himbeeck M and Nienhuijs S W 2017 Assessing the value of ehealth for bariatric surgery: the bepatient-trial technology and bariatric surgery Obes. Surg.

18. Kalarchian M A, Marcus M D, Courcoulas A P, Lutz C, Cheng Y and Sweeny G 2016 Structured dietary intervention to facilitate weight loss after bariatric surgery: A randomized, controlled pilot study Obesity

19. Tashakkori A and Teddlie C 2003 Handbook of mixed methods in social and behavioral research Sage Publ.

20. Creswell J W and Plano-Clark V L 2011 Choosing a mixed methods design Des. Conduct. Mix. Method Res.

21. Creswell J and Clark V P 2010 The foundations of mixed methods research Des. Conduct. Mix. methods Res.

22. Haute Autorité de Santé 2009 Obésité: prise en charge chirurgicale chez l'adulte Recommandations bonne Prat. Saint-Denis La ... 1-263 
23. Morana C, Collignon M and Nocca D 2018 Effectiveness of a Functional Rehabilitation Program After Bariatric Surgery: a Pilot Study Obes. Surg. 28 2321-6

24. Romain A J, Bernard P, Attalin V, Gernigon C, Ninot G and Avignon A 2012 Health-related quality of life and stages of behavioural change for exercise in overweight/obese individuals Diabetes Metab. $38352-8$

25. Glaser B . y A . S ( 1967 ). 1967 The discovery of grounded theory: strategies for qualitative research . New York: Aldine Publishing Company, Capítulo 3: " El muestreo teórico ", pp . 45-77 . Fac, Buenos Aires Soc. Ciencias Dom, Errandonea-Modalidad Infesta Traducci, Soc. I I Edici, Floreal Forni Pozzi, Llanos

26. Sarwer D B, Wadden T A and Fabricatore A N 2005 Psychosocial and behavioral aspects of bariatric surgery Obes. Res.

27. Sarwer D B, Dilks R J and West-Smith L 2011 Dietary intake and eating behavior after bariatric surgery: Threats to weight loss maintenance and strategies for success Surg. Obes. Relat. Dis.

28. Castro F G, Kellison J G, Boyd S J and Kopak A 2010 A methodology for conducting integrative mixed methods research and data analyses J. Mix. Methods Res.

\section{Tables}

Table 1:

Patients' characteristics at inclusion

\begin{tabular}{|lllll|}
\hline Variable & & $\begin{array}{l}\text { TelePhys group } \\
\mathbf{N}=\mathbf{4 5}\end{array}$ & $\begin{array}{l}\text { TeleDiet group } \\
\mathbf{N}=\mathbf{4 5}\end{array}$ & $\begin{array}{l}\text { Overall } \\
\mathbf{N}=\mathbf{9 0}\end{array}$ \\
\hline Age & Mean \pm SD & $41.9 \pm 9.1$ & $39.4 \pm 11.4$ & $40.6 \pm 10.4$ \\
\hline Women & $\mathrm{N}(\%)$ & $38(84.4 \%)$ & $35(77.8 \%)$ & $73(81.1 \%)$ \\
\hline BMI (kg/m2) & Mean \pm SD & $42.7 \pm 5.2$ & $44.0 \pm 6.0$ & $43.4 \pm 5.6$ \\
\hline Weight $(\mathrm{kg})$ & Mean \pm SD & $116.5 \pm 15.8$ & $124.0 \pm 16.6$ & $120.3 \pm 16.5$ \\
\hline & Gastric lap band & $8(17.8 \%)$ & $9(20 \%)$ & $17(18.9 \%)$ \\
\hline Types of surgey & Gastric bypass & $31(68.9 \%)$ & $31(68.9 \%)$ & $62(68.9 \%)$ \\
\hline & Sleeve & $6(13.3 \%)$ & $5(11.1 \%)$ & $11(12.2 \%)$ \\
\hline
\end{tabular}

BMI: Body mass Index; SD: standard deviation. BMI and weight were preoperative data. 
Table 2:

Numbers of steps during the 14-day period of monitoring at the first and sixth postoperative months in the TelePhys and TeleDiet groups

\begin{tabular}{|lllll|}
\hline Variable & \multicolumn{2}{c}{ TelePhys group } & TeleDiet group & P value $^{*}$ \\
\hline Number of steps at M1 & Mean \pm SD & $1073.10^{2} \pm 471.10^{2}$ & $839.10^{2} \pm 437.10^{2}$ & $\mathbf{0 . 0 4 5}$ \\
\cline { 2 - 5 } & N & 31 & 32 & \\
\hline Numbers of steps at M6 & Mean \pm SD & $1322.10^{2} \pm 523.10^{2}$ & $1340.10^{2} \pm 623.10^{2}$ & 0.915 \\
& N & 23 & 20 & \\
\hline Delta M6 - M1 & Mean \pm SD & $142.10^{2} \pm 535.10^{2}$ & $338.10^{2} \pm 512.10^{2}$ & 0.240 \\
\hline & N & 23 & 20 & \\
\hline P value** & & 0.250 & $\mathbf{0 . 0 1 0}$ & \\
\hline
\end{tabular}

M1: first month; M6: sixth month; SD: standard deviation; *: Between group comparison; **: Intra-group comparison.

Table 3:

Evolution of body weight monitored with the connected weight scale at the third and sixth postoperative months in the TelePhys and TeleDiet groups

\begin{tabular}{|lllll|}
\hline Variable & & TelePhys group & TeleDiet group & P-value* \\
\hline Weight M0 (Kg) & Mean \pm SD & $116.5 \pm 16$ & $124.0 \pm 16$ & 0.033 \\
& $\mathrm{~N}$ & 45 & 45 & \\
\hline Weight M3 (Kg) & Mean \pm SD & $98.8 \pm 16$ & $101.6 \pm 17$ & 0.523 \\
\hline & $\mathrm{N}$ & 28 & 28 & \\
\hline Weight M6 (Kg) & Mean \pm SD & $86.8 \pm 16$ & $89.4 \pm 15$ & 0.500 \\
\hline & $\mathrm{N}$ & 31 & 24 & \\
\hline Delta M6-M0 & Mean \pm SD & $-29.5 \pm 12.1$ & $-31.3 \pm 8.6$ & 0.541 \\
\hline & $\mathrm{N}$ & 31 & 24 & \\
\hline P value** & & $<0.0001$ & $<0.0001$ & \\
\hline
\end{tabular}

M0: preoperative; M3: third month; M6: sixth month; SD: standard deviation; *: Between group comparison; **: Intra-group comparison. 
Table 4:

Evolution of the health-related quality of life between the inclusion and the sixth postoperative month in the TelePhys and TeleDiet groups using the Quality Of Life, Obesity and Dietetics (QOLOD) scale

\begin{tabular}{|lllll|}
\hline Delta M6 - M1 & & $\begin{array}{l}\text { TelePhys group } \\
\text { N=21 }\end{array}$ & $\begin{array}{l}\text { TeleDiet group } \\
\text { N=21 }\end{array}$ & P-value* \\
\hline Physical impact & Mean \pm SD & $20.76 \pm 12.54$ & $24.19 \pm 16.63$ & 0.455 \\
\hline Psychosocial impact & Mean \pm SD & $15.95 \pm 19.27$ & $14.95 \pm 20.06$ & 0.870 \\
\hline Sex life & Mean \pm SD & $11.90 \pm 19.78$ & $5.48 \pm 23.39$ & 0.278 \\
\hline Comfort with food & Mean \pm SD & $8.19 \pm 25.41$ & $15.24 \pm 21.34$ & 0.229 \\
\hline Dieting experience & Mean \pm SD & $13.14 \pm 21.58$ & $10.67 \pm 19.89$ & 0.792 \\
\hline
\end{tabular}

M1: first month; M6: sixth month; SD: standard deviation; *: Between group comparison

\section{Figures}




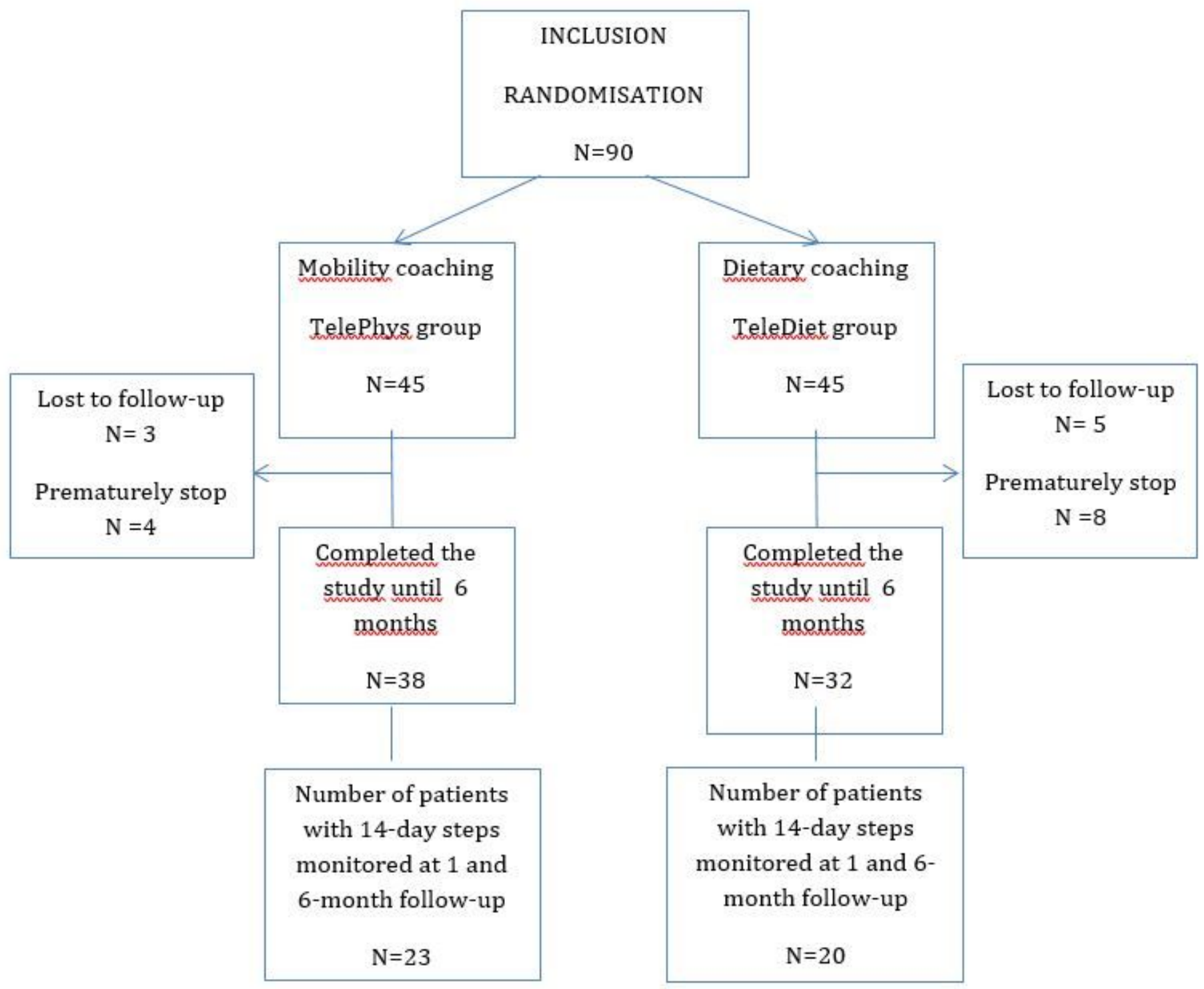

Figure 1

Flow chart of the MyGoodTrip randomized trial 

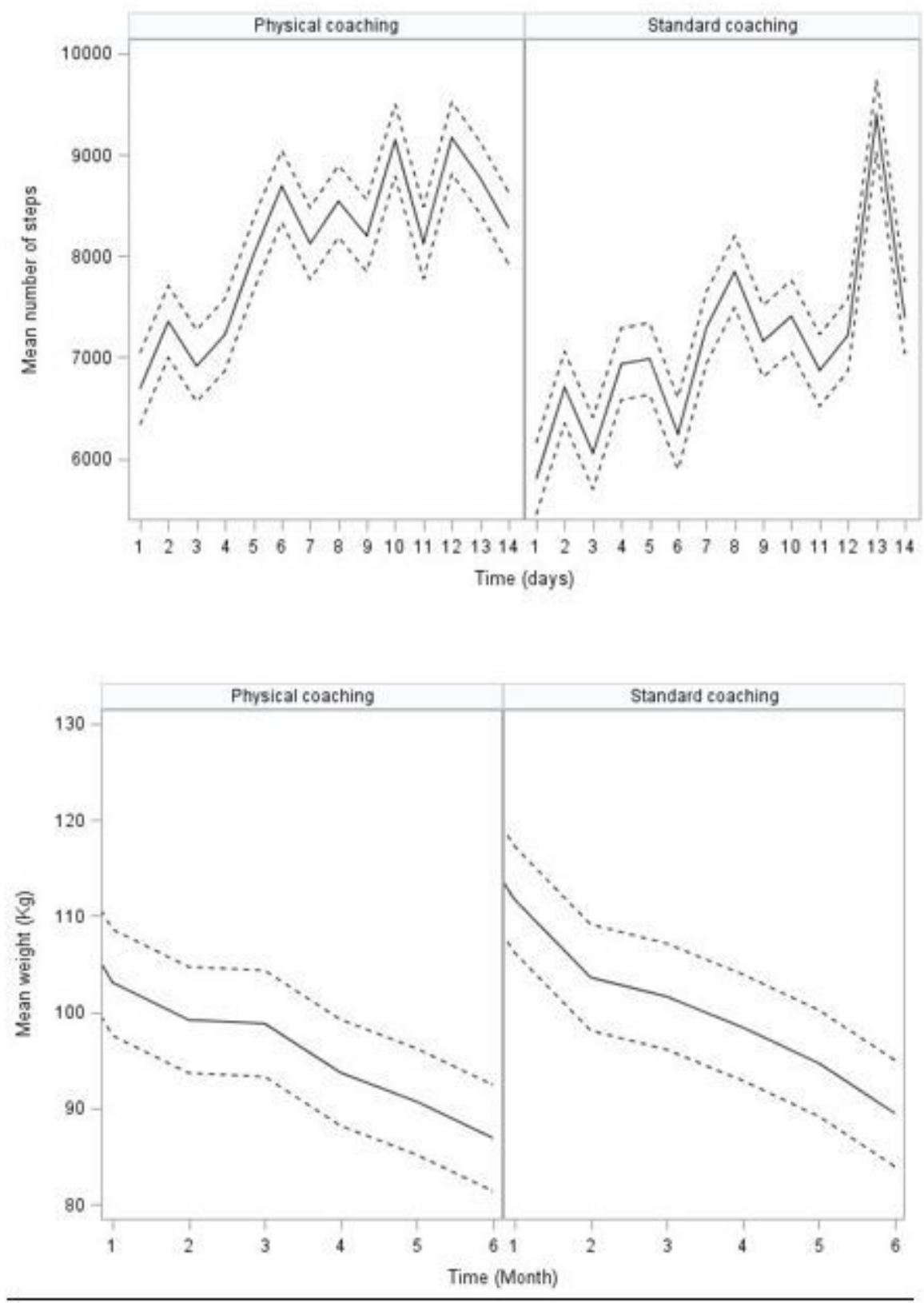

\section{Figure 2}

Mean number of day steps and mean weight evaluated with the connected devices during the 6 months in the TelePhys and TeleDiet groups steps 